\title{
The Stress and Reliability Analysis of HTR's Graphite Component
}

\author{
Xiang Fang, Haitao Wang, and Suyuan Yu \\ Institute of Nuclear and New Energy Technology, Tsinghua University, Beijing 100084, China \\ Correspondence should be addressed to Xiang Fang; fangxiang@tsinghua.edu.cn
}

Received 15 November 2013; Revised 6 February 2014; Accepted 7 February 2014; Published 18 March 2014

Academic Editor: Luciano Burgazzi

Copyright (C) 2014 Xiang Fang et al. This is an open access article distributed under the Creative Commons Attribution License, which permits unrestricted use, distribution, and reproduction in any medium, provided the original work is properly cited.

\begin{abstract}
The high temperature gas cooled reactor (HTR) is developing rapidly toward a modular, compact, and integral direction. As the main structure material, graphite plays a very important role in HTR engineering, and the reliability of graphite component has a close relationship with the integrity of reactor core. The graphite components are subjected to high temperature and fast neutron irradiation simultaneously during normal operation of the reactor. With the stress accumulation induced by high temperature and irradiation, the failure risk of graphite components increases constantly. Therefore it is necessary to study and simulate the mechanical behavior of graphite component under in-core working conditions and forecast the internal stress accumulation history and the variation of reliability. The work of this paper focuses on the mechanical analysis of pebble-bed type HTR's graphite brick. The analysis process is comprised of two procedures, stress analysis and reliability analysis. Three different creep models and two different reliability models are reviewed and taken into account in simulation. The stress and failure probability calculation results are obtained and discussed. The results gained with various models are highly consistent, and the discrepancies are acceptable.
\end{abstract}

\section{Introduction}

The high temperature gas cooled reactor is one of the candidate reactor types of the fourth generation advanced nuclear reactor [1]. At present, the development of pebblebed type HTR technology is oriented to modularization, miniaturization, and integration. The side reflective of the reactor core is piled up by a large number of graphite bricks. The reliability of side reflector graphite component impacts the integrity and safety of reactor core directly. This paper mainly focuses on the analysis and study of graphite component's mechanical behaviors under in-core working conditions. The graphite components are subjected to high temperature load and fast neutron irradiation load simultaneously when the reactor operates. High temperature and irradiation not only create significant internal stresses, but also change the physical properties of graphite in evidence. The graphite component might deteriorate into failure if the internal stress accumulates to a certain extent. Most of graphite properties significantly change in high temperature and neutron irradiation conditions, and the changing of properties makes the behavior of graphite component more complex [2]. Meanwhile, creep also plays a decisive role in the process. The majority of internal stresses can be relaxed by creep [3].

The mechanical analysis procedure of graphite component is sketched in Figure 1. The process consists of two steps. The first is stress analysis. The parameters of graphite (including Young's modulus, Poisson's ratio, the coefficient of thermal expanding, the irradiation-induced deformation, and the thermal conductivity), the geometric parameters (as the geometric model of graphite brick and finite element meshing), and the environment parameters (i.e., the boundary conditions for calculation, including temperature distribution and neutron dose distribution) are used as input data. Stress distribution and development history can be obtained after finite element analysis (FEA) calculation. Establishing a practical constitutive law is the key for this step. The thermal strain, irradiation-induced strain, and creep strain must be considered in the constitutive law. The second step is reliability analysis. In this step, the stress distribution gained in the first step is used as source data, and the reliability is estimated by form of failure probability or service life of graphite component [4]. Both deterministic and probabilistic methods 


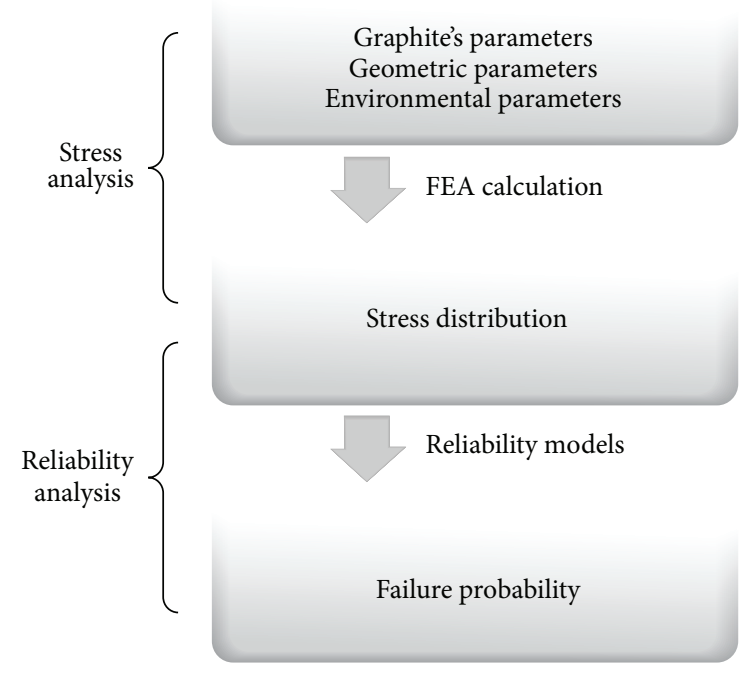

FIGURE 1: Mechanical analysis procedures of graphite component.

have been accepted in engineering standards and codes, but the probabilistic method is commonly regarded as the more suitable tool to evaluate the mechanical behaviors of brittle materials like graphite, due to the failure of brittle materials usually occurring within a certain range of stress rather than a specific stress value (i.e., the intensity value) $[5,6]$. Two kinds of probabilistic reliability models, the Weibull model and the Burchell model, are reviewed in the present paper.

A MSC.MARC based finite element code INET-GRA3D has been established in INET, Tsinghua University, to numerically study the mechanical behavior of graphite component in HTRs [7]. A candidate design of pebble-type HTR's graphite brick is selected to develop a three-dimensional finite element geometric model. Both analysis steps are implemented in the code. Various creep models and reliability models are contained in order to compare the results. In Section 2 of this paper, the constitutive law and creep models are reviewed and the main formulae of three graphite creep models are introduced. In Section 3, both Weibull model and Burchell model are reviewed in detail. In Section 4, the geometric model, boundary conditions, and initial data are summarized. Two kinds of graphite, ATR-2E and H-451, are selected as demonstration materials for HTR side reflector since their calculation-required original data are comprehensive. The results of different graphite types are calculated with different models. The results, analysis, and model assessment are given in Section 5. Stress distributions, stress history, and the corresponding failure probabilities are calculated and compared. In Section 6, there are the conclusions.

\section{Review of Constitutive Law and Creep Models}

2.1. The Constitutive Law. The constitutive law of nuclear graphite is [8]

$$
\sigma=D(T, \gamma) \varepsilon^{E}=D(T, \gamma)\left(\varepsilon-\varepsilon^{T}-\varepsilon^{R}-\varepsilon^{C}\right),
$$

where $\sigma$ is the stress tensor and $D(T, \gamma)$ is the elastic matrix as a function of temperature $T$ and neutron dose $\gamma$. The total strain tensor $\varepsilon$ is composed of the elastic strain $\varepsilon^{E}$, the thermal strain $\varepsilon^{T}$, the irradiation-induced strain $\varepsilon^{R}$, and the creep strain $\varepsilon^{C}$. The incremental form of (1) is

$$
d \sigma=D(T, \gamma) \cdot\left(d \varepsilon-d \varepsilon^{T}-d \varepsilon^{R}-d \varepsilon^{C}\right)+\varepsilon^{E} \cdot d D(T, \gamma),
$$

and $d \varepsilon^{T}$ is simplified and expressed as $\alpha(T, \gamma) d T$ in calculation, where $\alpha$ is the coefficient of thermal expansion (CTE) depending on $T$ and $\gamma \cdot d \varepsilon^{R}$ is obtained by experimental data of dimensional change rate. Equation (2) is used in finite element calculation.

2.2. Creep Models. The creep phenomenon greatly impacts the behavior of graphite under high temperature and neutron irradiation conditions. With the accumulation of neutron irradiation dose, the internal stresses of graphite component grow rapidly, making the failure probability increase significantly. However, most of the internal stress can be released by the action of creep. In the past few decades, researchers set up several mechanical models to describe creep behavior. Some models are based on fitting experimental data (as the linear viscoelastic model and the M2 model), some are approximation or correction of some other model (as the UKAEA model and the Kennedy model), and others are set up with microscopic theory (as the Kelly-Burchell model) [3]. In the present paper, the linear viscoelastic model, the UKAEA model, and the Kennedy model are reviewed, applied in simulation, and assessed.

2.2.1. Linear Viscoelastic Model. The creep behavior is always divided into two stages: the primary creep with strain $\varepsilon^{P C}$ and the secondary creep with strain $\varepsilon^{S C} \cdot \varepsilon^{P C}$ is transient creep strain, following a law of exponential growth. The secondary creep is a steady stage with a constant creep strain $\varepsilon^{S C}$. The total creep strain $\varepsilon^{C}$ in (1) is

$$
\varepsilon^{C}=\varepsilon^{P C}+\varepsilon^{S C}=\frac{a \sigma}{E_{0}}[1-\exp (-b \gamma)]+k \sigma \gamma,
$$

where $a$ and $b$ are constants. The saturated value of $\varepsilon^{P C}$ is always same with elastic strain $\varepsilon^{E}$; this indicates $a=1$ [9]. $k$ is the secondary creep coefficient, which is related to $T$ and $\gamma$. Equation (3) is the expression of linear viscoelastic creep model; "linear" denotes that there is a linear relationship between $\varepsilon^{S C}$ and $\gamma$. In order to simplify the calculation, the true creep strain is represented as

$$
\varepsilon^{C}=\varepsilon^{P C}+\varepsilon^{S C}=\frac{\sigma}{E_{0}}+k \sigma \gamma
$$

It has been shown that (4) is consistent with experimental stress-strain curve [10]. The experimental data of $k$ is required in the viscoelastic model. The data is limited, which means the linear viscoelastic model is applicable only to a few types of graphite. However, in any case, the linear viscoelastic model is always the most intuitive method to reflect the experimental results. 
2.2.2. UKAEA Model. Both the UKAEA model (or called the UK model for short) and the Kennedy model use other parameters instead of $k$, in order to solve the problem that $k$ 's experiment data is insufficient. The viscoelastic model is adapted by the UKAEA model and the Kennedy model in two different ways: the UKAEA model by modifying $k$ with Young's modulus and the Kennedy model by using the percentage volume change. Some experimental results show that Young's modulus $E$ and $k$ have approximate inversely proportional relation. The secondary creep coefficient $k$ at a neutron dose $\gamma$ can be given by

$$
k(T, \gamma)=k_{p}\left[\frac{E_{p}}{E(T, \gamma)}\right]=\frac{k_{p}}{S(T, \gamma)} .
$$

$E_{p}$ and $k_{p}$ are Young's modulus and creep coefficient in some specific region, respectively [11]. Their possible values are selected at neutron dose point $1 \times 10^{21} \mathrm{n} \cdot \mathrm{cm}^{-2}$ (EDN). $S(T, \gamma)$ is Young's modulus structure factor given by $E(T, \gamma) / E_{p}$. Equation (4) can be rewritten as

$$
\varepsilon^{C}=\varepsilon^{P C}+\varepsilon^{S C}=\frac{\sigma}{E_{0}}+k_{p} \int_{0}^{\gamma} \frac{\sigma}{S(T, \gamma)} d \gamma
$$

2.2.3. Kennedy Model. The percentage volume change $\Delta V / V$ of graphite is used as the substitution parameter in the Kennedy model [12]. $k$ is given as

$$
k(T, \gamma)=k_{p}\left[1-\mu \frac{(\Delta V / V)(T, \gamma)}{(\Delta V / V)_{m}}\right] \text {. }
$$

Under fast neutron irradiation, the volume of graphite shrinks first and expands later. $(\Delta V / V)_{m}$ in (7) stands for the maximum volume shrinking. $\mu$ is an empirical constant and varies with graphite grade. In this paper, $\mu=0.75$ [3]. Equation (4) can be rewritten as

$$
\varepsilon^{C}=\varepsilon^{P C}+\varepsilon^{S C}=\frac{\sigma}{E_{0}}+k_{p} \int_{0}^{\gamma}\left[1-\mu \frac{(\Delta V / V)(T, \gamma)}{(\Delta V / V)_{m}}\right] \cdot \sigma d \gamma .
$$

\section{Review of Reliability Models}

The failure probability is usually used to express the reliability of graphite component. A graphite brick is divided into many integration points in the FEM calculation, and the total failure probability is a summation of all integration points' failure probabilities. There are mainly two completely different ideas to study the failure behavior of graphite: one is based on fitting experimental results of unirradiated graphite specimens (as the Weibull model), and the other proceeds from the microscopic mechanism of graphite rupture and connects the microcrack of crystal lattice with macrofailure (as the Burchell model).

The destructive testing of graphite specimen shows that there is an $S$-shape relationship between the failure probability and the tensile stress. Figure 2 is the tensile destruction curve of H-451 graphite, an extrusion forming nuclear

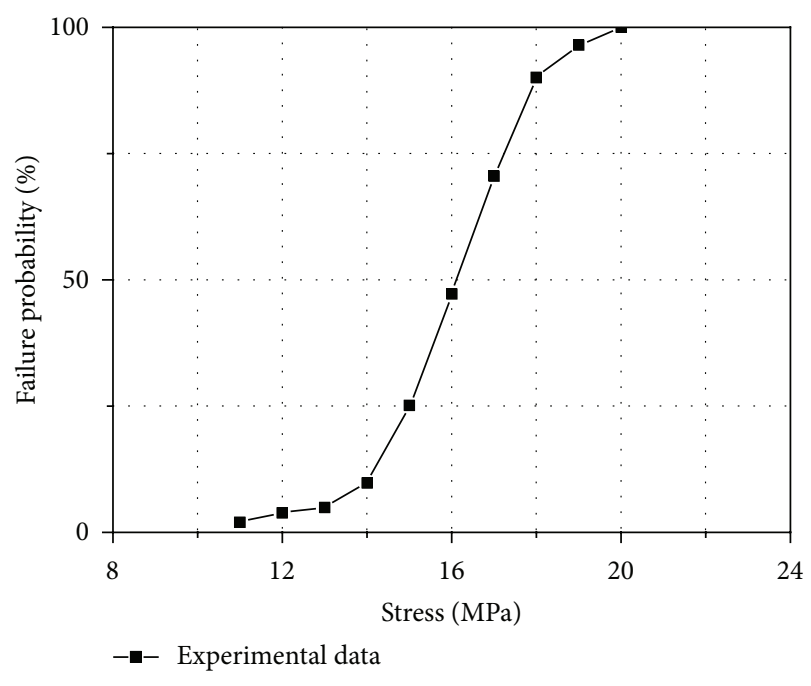

FIgUre 2: Tensile destruction curve of $\mathrm{H}-451$ graphite.

graphite. The failure probability increases from 0 to $100 \%$ when the stress rises from $10 \mathrm{MPa}$ to $20 \mathrm{MPa}$. The stress corresponding to $50 \%$ failure probability, $16 \mathrm{MPa}$, is always defined as the mean strength point.

3.1. Weibull Model. The fitting curve method is used in Weibull model. The failure probability $P_{i}$ at number $i$ integration point of graphite brick can be designated as

$$
P_{i}=1-\exp \left[-\left(\frac{\sigma}{S_{c}}\right)^{m}\right]
$$

where $m$ and $S_{c}$ are two Weibull parameters. $m$ is a shape parameter and $S_{c}$ is the characteristic strength value. Both parameters arise from the fitting experimental curves like Figure 2. With logarithmic transformation, (9) changes into the form of

$$
\ln \left(-\ln \left(1-P_{i}\right)\right)=-m \cdot \ln \sigma+m \cdot \ln S_{c},
$$

corresponding to the straight line equation $y(x)=a x+b$. $1-P_{i}$ indicates the survival probability of graphite specimen. Thus, the parameters $\left(m, S_{c}\right)$ can be obtained by fitting experimental data $\left(\sigma, P_{i}\right)$.

The survival probability of the graphite component is

$$
P_{s}=\exp \left\{-\sum_{i}\left[\left(\frac{\sigma}{S_{c}}\right)^{m} \times \frac{V_{i}}{V}\right]\right\},
$$

where $V_{i}$ is the representative volume of integration point $i$ and $V$ is the volume of graphite component; $V=\sum_{i} V_{i}$. The failure probability of the graphite component is then expressed as [13]

$$
P_{\text {tot }}=1-P_{s}=1-\exp \left\{-\sum_{i}\left[\left(\frac{\sigma}{S_{c}}\right)^{m} \times \frac{V_{i}}{V}\right]\right\} .
$$

As the two parameters $m$ and $S_{c}$ are contained, (12) is known as the two-parameter Weibull model, which is used 
in the German HTR code draft KTA-3232 [14] to generate a value of failure probability for graphite components in HTRs. Another Weibull model, called three-parameter Weibull model, has been proposed in ASME code 2010, HHA-II-3000 [15]. The failure probability $P_{i}$ in (9) is modified as

$$
P_{i}=1-\exp \left[-\left(\frac{\sigma-S_{0}}{S_{c}-S_{0}}\right)^{m}\right]
$$

where the third parameter $S_{0}$ is called the threshold parameter, representing the stress value corresponding to low failure probability in Figure 2. The failure probability of the graphite component is

$$
P_{\text {tot }}=1-P_{s}=1-\exp \left\{-\sum_{i}\left[\left(\frac{\sigma-S_{0}}{S_{c}-S_{0}}\right)^{m} \times \frac{V_{i}}{V}\right]\right\} .
$$

However, only two-parameter Weibull model will be used in this paper's calculation because of the lack of $S_{0}$ 's experimental data.

3.2. Burchell Model. The Burchell model, which is founded from the microfracture mechanism of graphite, is an evolution of the Rose and Tucker fracture model which was developed in the 1970s [5]. It has been verified that the Burchell model is able to simulate the tensile test results in Figure 2 very well [13].

Each integration point of graphite FEM model is regarded as comprising of a pile of graphite particles with magnitude $\mathrm{mm}$ or even less. The particle is so small that the anisotropy of graphite's microstructure is expressed. Each particle contains a so-called "weakness plane" which parallels the $a$-axis of the particle (parallel to the layer of carbon atoms) and is oriented at angle $\theta$ to the fracture exposure. The starting points of microstructural fracture, a great deal of pores, are randomly distributed in the graphite particle matrix. Cracks extend by tearing the particles on their way along with the weakness planes. A graphite particle matrix which contains particles and pores is shown in Figure 3. $a$ and $c$ are the mean filler particle size and pore radius, respectively. When stressed at a level $\sigma$, a particle's fracture probability along the weakness plane is [16]

$$
P_{i}=\frac{4}{\pi} \cos ^{-1}\left(\frac{K_{I c}}{K_{I}}\right)^{1 / 3}
$$

where $K_{I c}$ is known as the critical stress intensity factor which reflects the resistance capacity of a particle against cracks. $K_{I}$ is a stress intensity factor associated with a pore. $K_{I}$ is defined as

$$
K_{I}=\sigma \sqrt{\pi c}
$$

The limits of particle fracture probability in (15) must be defined as follows: when $K_{I} \leq K_{I c}, P_{i}=0$, the particle cannot be broken; when $K_{I} \geq 2 \sqrt{2} K_{I c}, P_{i}=1$, the particle will certainly break; when $K_{I c} \leq K_{I} \leq 2 \sqrt{2} K_{I c}$, the corresponding probability of fracture is $P_{i}$.

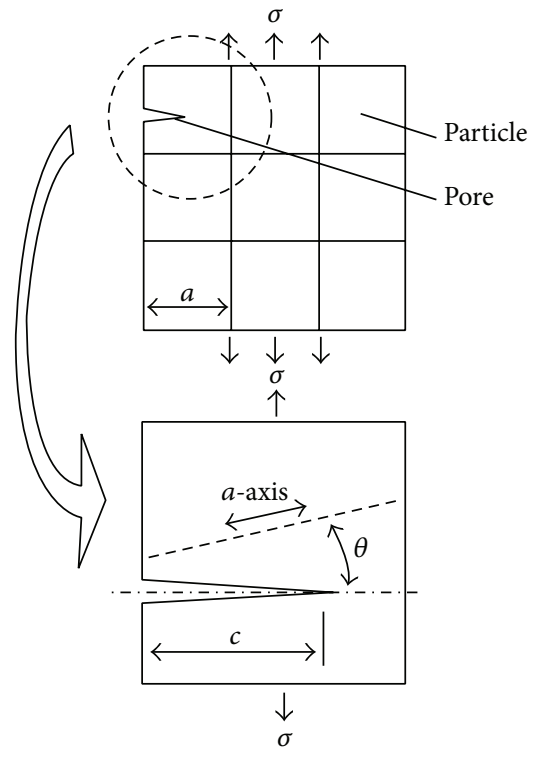

FIGURE 3: A graphite particle matrix containing particles and pores.

If there are $n$ particles along the crack width row (or depth for Figure 3), the failure probability of the row is

$$
P_{n}=\left(P_{i}\right)^{n}=\left[\frac{4}{\pi} \cos ^{-1}\left(\frac{K_{I c}}{\sigma \sqrt{\pi c}}\right)^{1 / 3}\right]^{n}
$$

where $n$ can be regarded as $n=b / a$ and $b$ is the representative size of integration point. Equation (17) indicates the chance for the crack to move forward a length $a$. The crack will extend only if each particle along the way of extending is torn. With the crack propagating, the crack size will increase from $c$ to $c+i a, i=1,2,3 \ldots$. The probability of crack extending $i$ rows of particles can be derived as

$$
P_{n}=\prod_{i=0}^{i}\left[\frac{4}{\pi} \cos ^{-1}\left(\frac{K_{I c}}{\sigma \sqrt{\pi(c+i a)}}\right)^{1 / 3}\right]^{b / a}
$$

where $a$ and $K_{I c}$ are constants for a particular kind of graphite, but $c$ inevitably is distribution data. There exist many initial pores in unirradiated graphite. The pores are considered to be the origin of cracks. In order to characterize the dispersion characterization of pore size, a probability function $f(c)$ is defined as

$$
f(c)=\text { const } \cdot \exp \left[-\frac{1}{2}\left(\frac{\ln 2 c-\ln S_{0}}{\ln S_{d}}\right)^{2}\right],
$$

where $S_{0}$ is the mean pore size and $S_{d}$ is a statistical parameter reflecting the spread of $c$ 's distribution. The probability that the size of a pore falls between $c$ and $c+d c$ is $f(c) d c$. The chance that a single pore tears an integration point under stress $\sigma$ is

$$
P_{f c}=\int_{0}^{\infty} f(c) \cdot P_{n}(\sigma, c) d c
$$


TABLE 1: Graphite type and model selection.

Graphite type

ATR-2E

H-451

Models selected for stress analysis

(1) Linear viscoelastic model

(2) UKAEA model $\quad$ (1) Kennedy model

(3) Kennedy model

Models selected for reliability analysis

(1) Two-parameter Weibull model

(1) Two-parameter Weibull model

(2) Burchell model

The survival probability of the integration point is

$$
P_{f s}=\left(1-P_{f c}\right)^{2}=\left[1-\int_{0}^{\infty} f(c) \cdot P_{n}(\sigma, c) d c\right]^{2} .
$$

As each pore has two sides, there is a square in the right side of (21). If $N$ is defined as the number of pores per unit volume and $V$ is the volume of integration point, $N V$ is the amount of pores in the integration point. The total fracture probability of the part is

$$
P_{f \text { tot }}=1-P_{s}^{N V}=1-\left[1-\int_{0}^{\infty} f(c) \cdot P_{n}(\sigma, c) d c\right]^{2 N V} .
$$

The graphite component possesses many integral parts whose volumes and stress levels are different. Therefore, the survival probability of the component can be derived by multiplying principle [17]:

$$
P_{s}=\prod_{j}\left[1-\int_{0}^{\infty} f(c) \cdot P_{n}(\sigma(j), c) d c\right]^{2 N V(j)} .
$$

And the total failure probability of the graphite brick is

$P_{\mathrm{tot}}=1-P_{s}=1-\prod_{j}\left[1-\int_{0}^{\infty} f(c) \cdot P_{n}(\sigma(j), c) d c\right]^{2 N V(j)}$.

Nine graphite parameters are required when calculating the reliability of graphite components with Burchell model, most of which are microparameters. The initial data of H-451 graphite will be given in Section 4 .

\section{Finite Element Calculation}

The finite element modeling of graphite brick, the boundary conditions, and some initial data of graphite will be described in this section. A three-dimensional finite element model is established according to a candidate design of the pebblebed HTR graphite side reflector. The FEM geometric model is simplified to a certain extent in order to reduce the amount of calculation.

4.1. FEM Model and Boundary Conditions. A symmetric FEM geometric model is shown in Figure 4, as well as the boundary condition of temperature distribution at the end

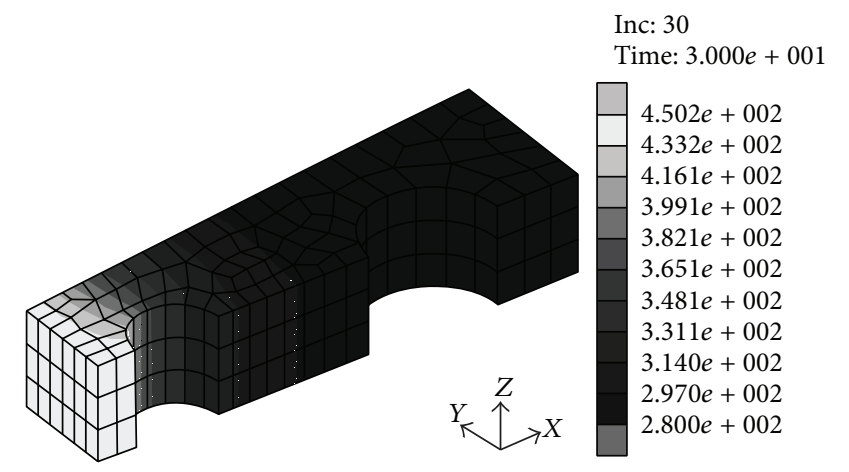

FIGURE 4: FEM model of graphite brick and temperature distribution at EOL (unit: ${ }^{\circ} \mathrm{C}$ ).

of 30 full power years' service life (EOL). The left side of the brick neighbors reactor core while the right side neighbors peripheral carbon bricks. The inner hole is for the control rod and the other hole serves as helium path. The temperature of the core side is $450^{\circ} \mathrm{C}$ while the outer side is $280^{\circ} \mathrm{C}$. Another boundary condition, the neutron dose, is also distributed nonuniformly. The maximum fast neutron dose at the core side reaches $1 \times 10^{22} \mathrm{n} \cdot \mathrm{cm}^{-2}(\mathrm{EDN})$ at EOL and decreases in radial direction of the core towards the outside, following an exponential law. The neutron dose unit EDN is the abbreviation of equivalent DIDO nickel dose. EDN has a transformation relation with another neutron dose unit dpa (displacement per atom) [3]:

$$
1 \times 10^{22} \mathrm{n} \cdot \mathrm{cm}^{-2}(\mathrm{EDN}) \cong 13 \mathrm{dpa} .
$$

4.2. Model Selection and Parameter Values. Two types of graphite, ATR-2E and H-451, are selected in this paper to simulate the behavior of graphite and validate the models. As different types of irradiation experiments have been carried out for various types of graphite, the resource data of different types of graphite is not the same. That indicates that not every kind of graphite can be simulated with all models. For example, it is unable to simulate the irradiation creep behavior of H-451 graphite brick with the viscoelastic model due to the lack of $k$ 's experimental data of H-451 graphite. The graphite types and corresponding calculation models are listed in Table 1 . The calculation of ATR-2E graphite focuses on the comparison of three different creep models, while the calculation of H-451 graphite focuses on the comparison of two different reliability models. The calculation results and model comparisons are summarized in Section 5. 
TABLE 2: Weibull parameters of ATR-2E and H-451 graphite.

\begin{tabular}{lcc}
\hline & \multicolumn{2}{c}{ Graphite type } \\
& ATR-2E & H-451 \\
\hline Weibull parameter, $m$ & 9.0 & 9.97 \\
Weibull parameter, $S_{c}(\mathrm{MPa})$ & 18.5 & 16.86 \\
\hline
\end{tabular}

TABLE 3: Burchell parameters of $\mathrm{H}-451$ graphite.

\begin{tabular}{lc}
\hline Parameter & Value \\
\hline Mean particle size, $a(\mu \mathrm{m})$ & 500 \\
Bulk density, $\rho\left(\mathrm{g} / \mathrm{cm}^{3}\right)$ & 1.79 \\
Mean pore size, $S_{0}(\mu \mathrm{m})$ & 42 \\
Statistical parameter, $S_{d}$ & 1.9 \\
Mean pore area, $\mathrm{Pa}\left(\mu \mathrm{m}^{2}\right)$ & 700 \\
Number of pores per $\mathrm{m}^{3}, N\left(\mathrm{~m}^{-3}\right)$ & $2.97 \times 10^{8}$ \\
Integration point volume, $V\left(\mathrm{~m}^{3}\right)$ & Real-time computed \\
Specimen breadth, $b(\mathrm{~mm})$ & Real-time computed \\
Particle critical stress intensity factor, $K_{I c}$ & 0.285 \\
$\left(\mathrm{MPa} \cdot \mathrm{m}^{1 / 2}\right)$ & \\
\hline
\end{tabular}

The two Weibull parameters of graphite are obtained by tensile test. The values for ATR-2E and H-451 are shown in Table 2.

The Burchell parameters of $\mathrm{H}-451$ graphite are shown in Table 3, including all nine parameters required. Some of the parameter values change after neutron irradiation, and the variation and new values are induced in [16]. It is worth noting that the value of $K_{I c}$ rises to $0.42 \mathrm{MPa} \cdot \mathrm{m}^{1 / 2}$ at a low dose level $10^{20} \mathrm{n} \cdot \mathrm{cm}^{-2}(\mathrm{EDN})$.

\section{Results and Comparisons}

The internal stress distribution of graphite brick at EOL is shown in Figure 5 as an example. The result shown in Figure 5 is obtained with ATR-2E graphite and the linear viscoelastic model. The stress distribution and concentration areas gained by other cases are the same. The stress concentrates in two areas in each case, and the stress concentrative points $\mathrm{A}$ and $\mathrm{B}$ are selected to analyze the stress history.

5.1. Results of ATR-2E Graphite. The stress history of points A and $B$ is shown in Figure 6. The stress development processes of two stress concentrative points are completely different. The stress on point A slightly increases and then decreases to initial value in the first 15 years and rises rapidly thereafter. The maximum stress value appears at EOL and reaches about $6 \mathrm{Mpa}$. Although various kinds of raw data are used in different creep model calculations, the stress results are very close to each other. It is hard to tell which stress value among the three ones is higher from stress diagram of point $\mathrm{A}$.

On point $B$, the stress increases continually in most of graphite brick's service time but declined slightly near EOL. The peak value reaches $8-9 \mathrm{MPa}, 30 \%$ higher than peak value on point $\mathrm{A}$. The curves of various models do not intersect each other. It is quite clear that the Kennedy model gives

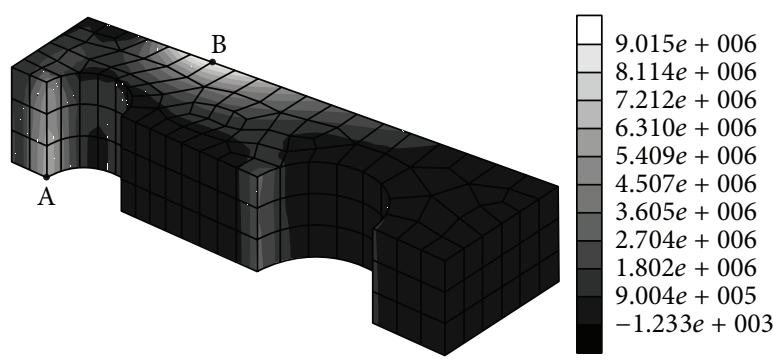

FIgURE 5: Stress distribution at EOL (unit: Pa).

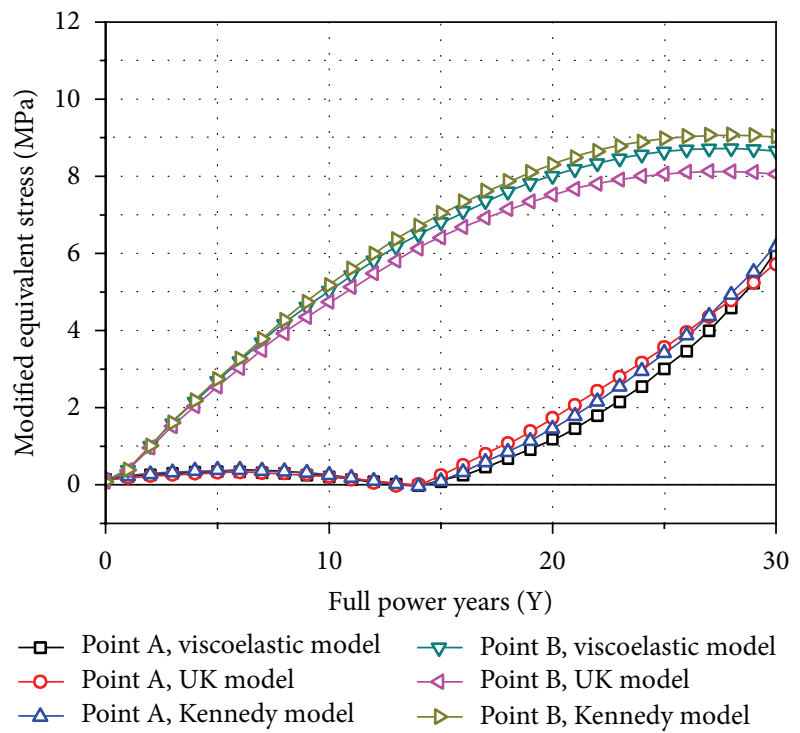

FIGURE 6: Stress history in service life of ATR-2E graphite brick.

the highest stress, while the UKAEA model gives the lowest. However, the model error is no more than $10 \%$, which will not negative the validity of any creep model.

Figure 7 shows the reliability analysis results of ATR-2E graphite brick. The failure probability rapidly grows in the first 20 years, followed by a decline near EOL. The failure probability calculated by the Kennedy model is the highest with peak value exceeding $10^{-7}$, while the failure probability by the UKAEA model is the lowest with peak value $4.5 \times$ $10^{-8}$. Different creep models not only give exactly the same failure probability change trend, but also present very close results. This means that each creep is effective and competent for engineering design. In addition, if one feels like getting the most conservative design, selecting the Kennedy creep model is recommended.

5.2. Results of $H-451$ Graphite. It is shown in the previous calculation that each of the three selected creep models is valid for design. Therefore, only the Kennedy creep model is adopted in the calculation of $\mathrm{H}-451$ graphite brick's mechanical behavior. The points A and B's internal stress development curves of H-451 graphite brick are shown in Figure 8. The changing process of stress on point A is very similar with that of ATR-2E graphite. The peak value is also $\sim 6 \mathrm{MPa}$, 


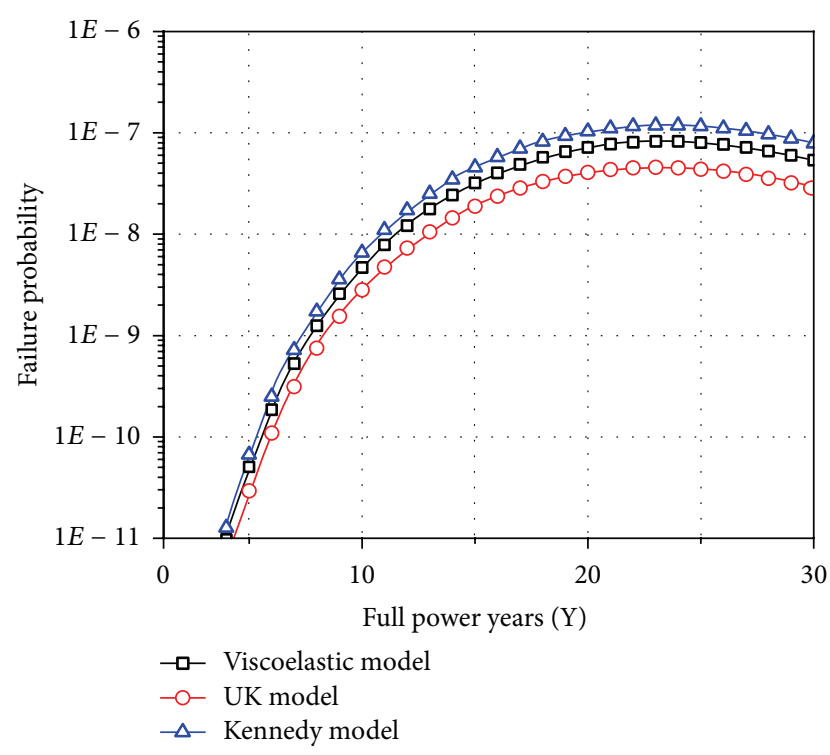

FIGURE 7: Failure probability history of ATR-2E graphite brick.

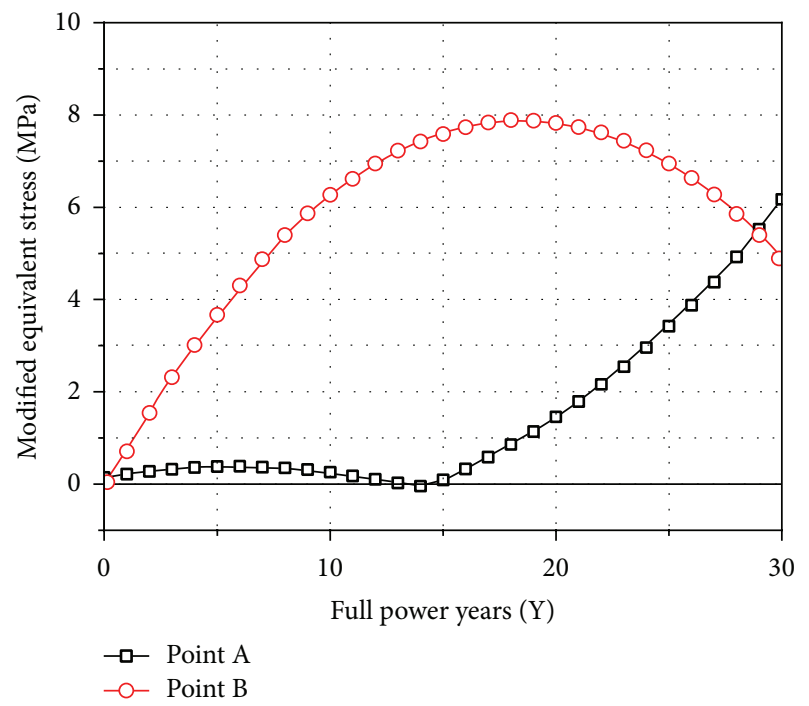

FIGURE 8: Stress history in service life of $\mathrm{H}-451$ graphite brick.

presenting at EOL. On point $B$, the stress peak value appears near 20 years' service time and reaches $8 \mathrm{MPa}$. The stress begins to fall since, and only $5 \mathrm{MPa}$ is left at EOL.

The subsequent reliability analysis' results are shown in Figure 9. The results of two-parameter Weibull model are very close to that of ATR-2E graphite. This means that the two kinds of graphite have almost the same reliability level. Although the establishing mechanisms of the two reliability models are completely different, their obtained results are pretty similar. Compared with the results of Weibull model, the failure probability obtained by Burchell model changes much faster. That indicates that the Burchell model is much more sensitive to stress (or neutron dose) than the Weibull model. The Burchell model's result is much lower in the beginning, but rises fast, and exceeds the Weibull model's

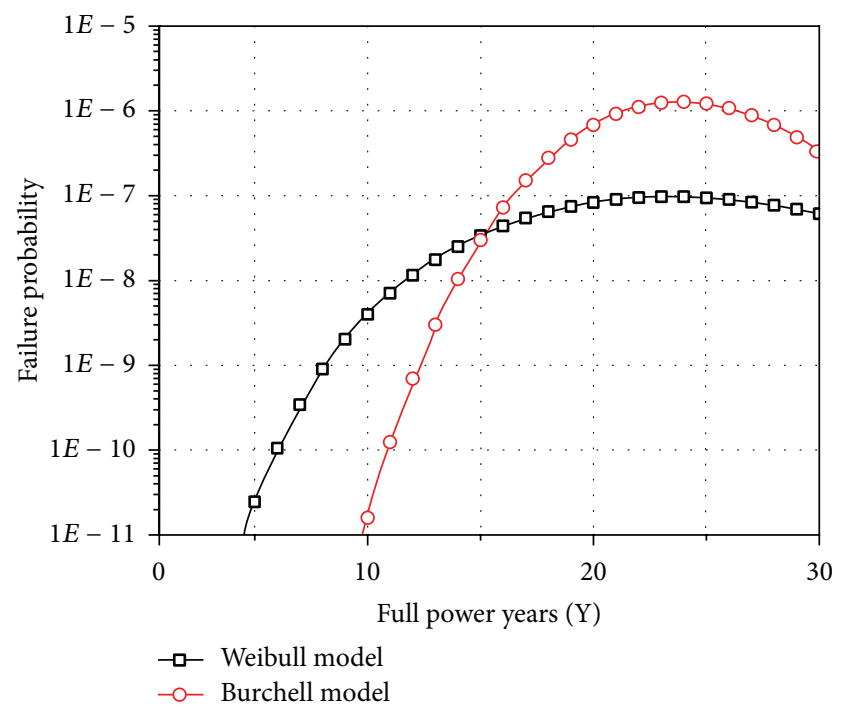

FIGURE 9: Failure probability history of H-451 graphite brick.

result at 15 years' service time. The peak value reaches $10^{-6}$, still far from the threshold value $10^{-4}$ which is determined in [15].

\section{Conclusions}

Along with the advance of technology, the HTR is oriented to modularization, miniaturization, integration, and other new development direction. The safety of reactor structural components is one of the most important focuses in the meantime. The reliability of graphite component is directly related to the integrity of the reactor core and affects the safety of the entire reactor. When the reactor is on operation, the graphite components are exposed to high temperature and fast neutron irradiation. The temperature and irradiation load will not only create an accumulation of stresses, but also dramatically change the nature of graphite. Creep will also influence the whole process of mechanics at the same time. A comprehensively constitutive model calculation, including elastic behavior, thermal strain, irradiation deformation, and creep phenomenon, must be established in order to properly simulate the mechanical behavior of graphite component and predict the internal stresses and failure probability.

Three creep models and two reliability models are reviewed, applied, and compared in the present paper. Two types of graphite, ATR-2E and $\mathrm{H}-451$, are selected as the demonstration material for calculation. The main conclusions of graphite component's mechanical analysis and model comparisons are provided. It is shown in the stress analysis procedure that all three creep models can be used for stress prediction and engineering design. The Kennedy model is the most conservative one. In the reliability analysis procedure, the results obtained by both reliability models are in satisfactory agreement and both much lower than the threshold value $10^{-4}$. The discrepancies on the specific values indicate that the Burchell model leads to the failure probability more sensitive to stress (or neutron dose) than that of the Weibull model. 
In addition, more models have been established but still lack for engineering design experience, as the Kelly-Burchell creep model and M2 creep model for stress analysis and the three-parameter Weibull model for reliability analysis. Other practical models are still being established as well. There is still much further work that can be carried out in terms of model application and assessment.

\section{Conflict of Interests}

The authors declare that there is no conflict of interests regarding the publication of this paper.

\section{Acknowledgments}

This project is supported by the Tsinghua University Initiative Scientific Research Program (no. 20111080959) and the Specialized Research Fund for the Doctoral Program of Higher Education (no. 20120002120010).

\section{References}

[1] T. Abram and S. Ion, "Generation-IV nuclear power: a review of the state of the science," Energy Policy, vol. 36, no. 12, pp. 43234330, 2008 .

[2] B. T. Kelly and T. D. Burchell, "The analysis of irradiation creep experiments on nuclear reactor graphite," Carbon, vol. 32, no. 1, pp. 119-125, 1994.

[3] T. D. Burchell, K. L. Murty, and J. Eapen, "Irradiation induced creep of graphite," JOM, vol. 62, no. 9, pp. 93-99, 2010.

[4] T. Oku and M. Ishihara, "Lifetime evaluation of graphite components for HTGRs," Nuclear Engineering and Design, vol. 227, no. 2, pp. 209-217, 2004.

[5] A. P. G. Rose and M. O. Tucker, "A fracture criterion for nuclear graphite," Journal of Nuclear Materials, vol. 110, no. 2-3, pp. 186195, 1982.

[6] T. Burchell, T. Yahr, and R. Battiste, "Modeling the multiaxial strength of H-451 nuclear grade graphite," Carbon, vol. 45, no. 13, pp. 2570-2583, 2007.

[7] H. Wang and S. Yu, "Uncertainties of creep model in stress analysis and life prediction of graphite component," Nuclear Engineering and Design, vol. 238, no. 9, pp. 2256-2260, 2008.

[8] D. K. L. Tsang and B. J. Marsden, "Constitutive material model for the prediction of stresses in irradiated anisotropic graphite components," Journal of Nuclear Materials, vol. 381, no. 1-2, pp. 129-136, 2008.

[9] T. D. Burchell, "Irradiation induced creep behavior of H-451 graphite," Journal of Nuclear Materials, vol. 381, no. 1-2, pp. 4654, 2008.

[10] X. Fang, H. Wang, S. Yu, and C. Li, "The various creep models for irradiation behavior of nuclear graphite," Nuclear Engineering and Design, vol. 242, pp. 19-25, 2012.

[11] B. T. Kelly and J. E. Brocklehurst, "UKAEA reactor group studies of irradiation-induced creep in graphite," Journal of Nuclear Materials, vol. 65, no. 1, pp. 79-85, 1977.

[12] C. R. Kennedy, W. P. Eatherly, and R. L. Senn, "Compressive creep characteristics of graphite under irradiation," in Proceedings of the 33rd Pacific Coast Regional Meeting of the American Ceramic Society, San Francisco, Calif, USA, October 1980.
[13] S. Y. Yu, X. Fang, H. T. Wang, and C. F. Li, "Failure probability study of HTR graphite component using microstructure-based model," Nuclear Engineering and Design, vol. 253, pp. 192-199, 2012.

[14] KTA-3232, "Keramische Einbauten in HTR-Reacktordruckbehälten," Sicherheitstechnische Regel des KTA, 1992.

[15] HHA-II-3000, "Rules for construction of nuclear facility components," ASME boiler and pressure vessel code, III, Division 5, 2010.

[16] T. D. Burchell, "A microstructurally based fracture model for polygranular graphites," Carbon, vol. 34, no. 3, pp. 297-316, 1996.

[17] M. Ishihara, T. Takahashi, and S. Hanawa, "Applicability of Advanced Design Method of Graphite Components by Microstructure-Based Brittle Fracture Model," SmiRT 16, Washington DC. Paper \#1920, 2001. 


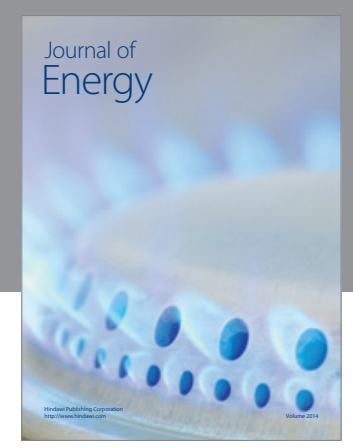

Journal of

Industrial Engineering
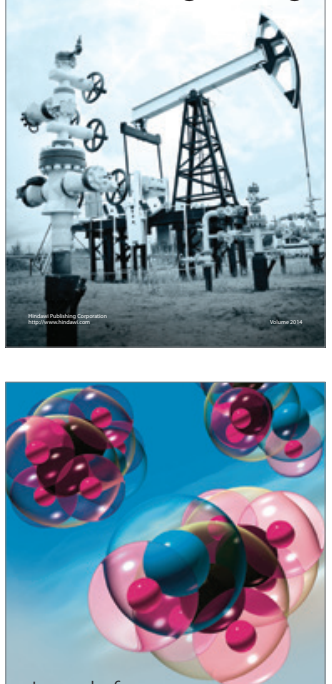

Fuels
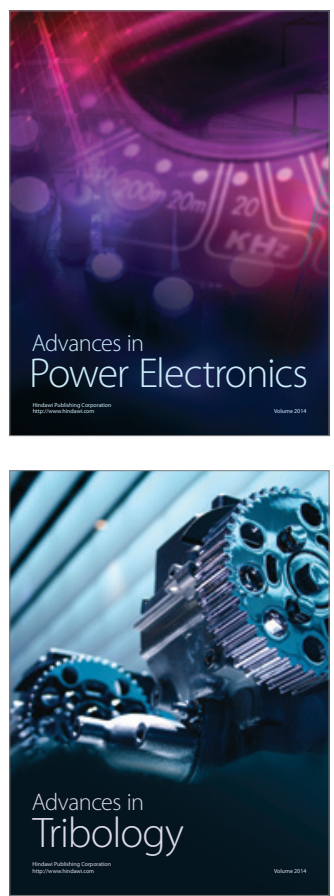

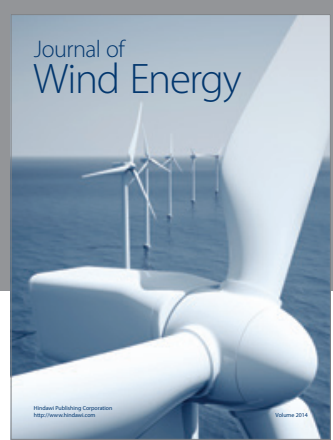

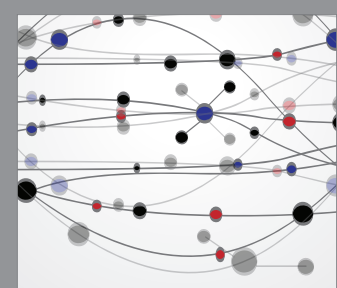

The Scientific World Journal

Submit your manuscripts at http://www.hindawi.com

Journal of

Structures
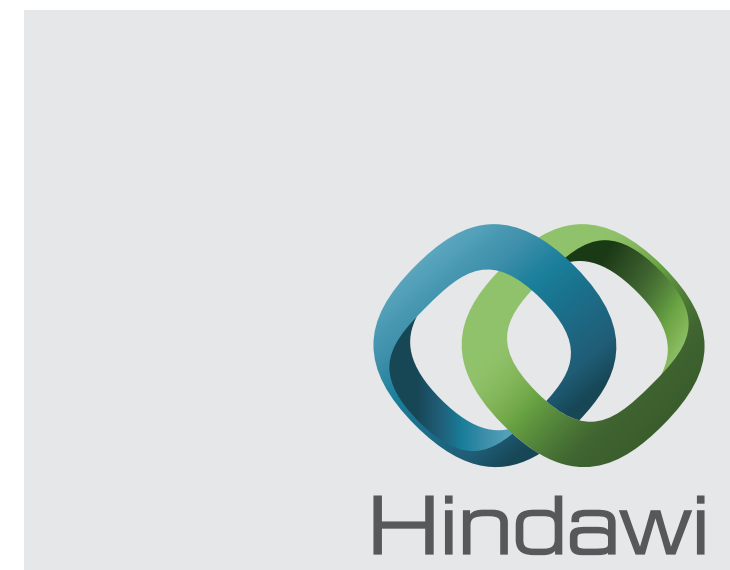

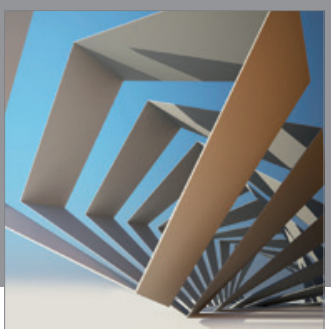

Rotating

Machinery
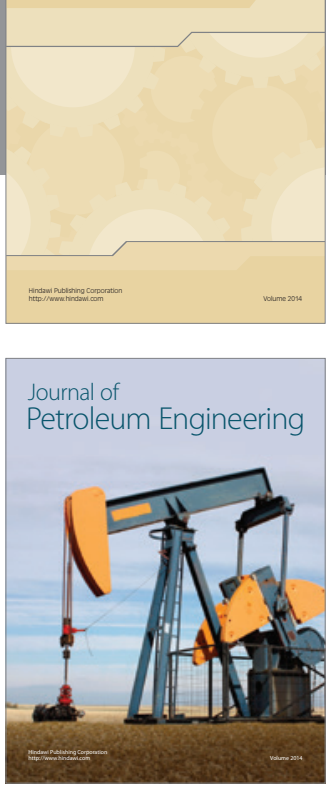

Journal of

Solar Energy
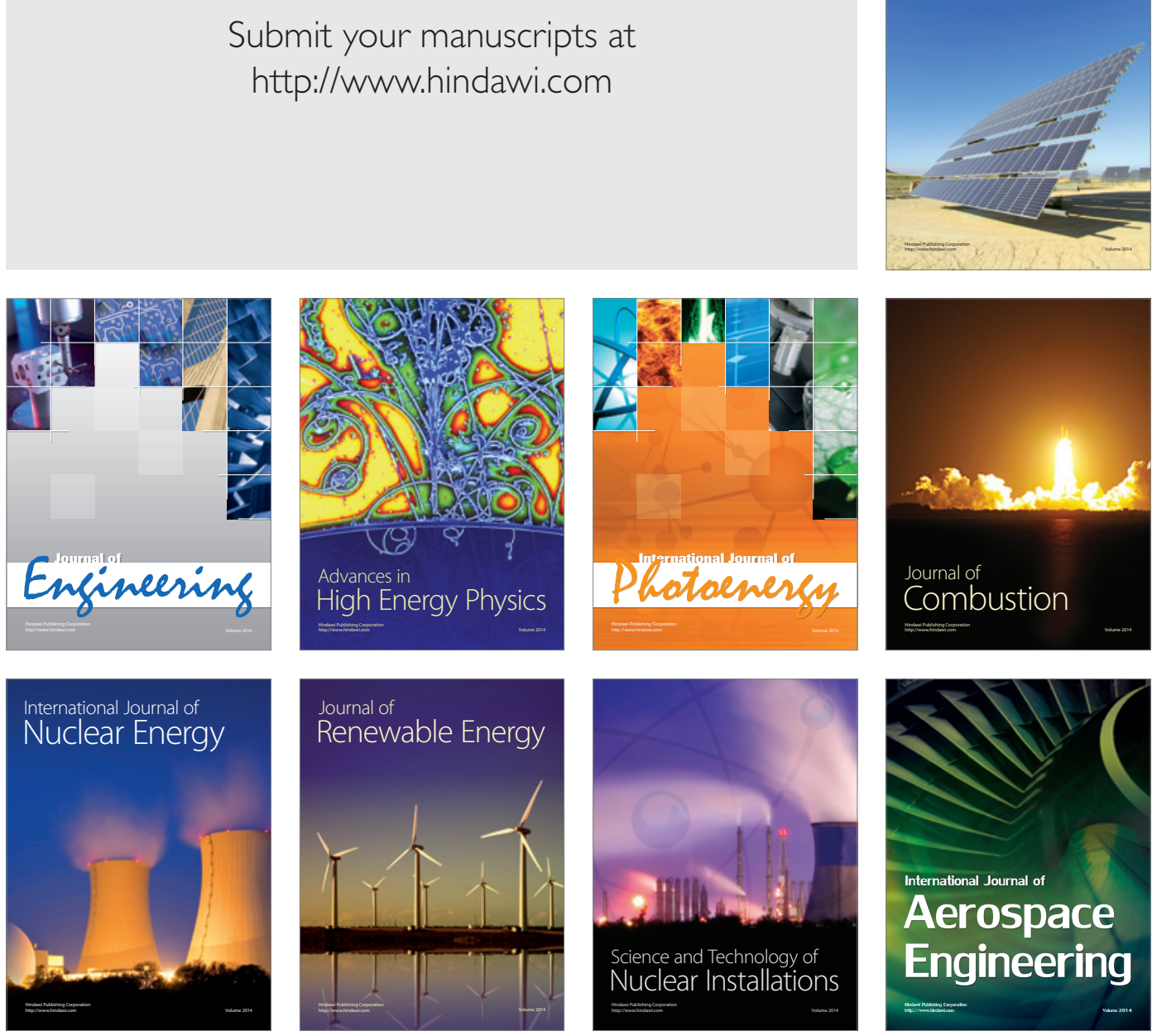